\title{
Event-by-Event Analysis of Baryon-Strangeness Correlations: Pinning Down the Critical Temperature and Volume of QGP Formation
}

\author{
Stephane Haussler ${ }^{1}$, Horst Stöcker ${ }^{1,2}$, and Marcus Bleicher ${ }^{2}$ \\ 1) Frankfurt Institute for Advanced Studies (FIAS), Johann Wolfgang Goethe Universität, \\ Max-von-Laue-Str. 1, 60438 Frankfurt am Main, Germany \\ 2) Institut für Theoretische Physik, Johann Wolfgang Goethe Universität, \\ Max-von-Laue-Str. 1, 60438 Frankfurt am Main, Germany
}

\begin{abstract}
The recently proposed baryon-strangeness correlation $\left(C_{B S}\right)$ is studied with a string-hadronic transport model (UrQMD) for various energies from $E_{\text {lab }}=4 \mathrm{AGeV}$ to $\sqrt{s}=200 \mathrm{AGeV}$. It is shown that rescattering among secondaries can not mimic the predicted correlation pattern expected for a Quark-Gluon-Plasma. However, we find a strong increase of the $C_{B S}$ correlation function with decreasing collision energy both for $\mathrm{pp}$ and $\mathrm{Au}+\mathrm{Au} / \mathrm{Pb}+\mathrm{Pb}$ reactions. For $\mathrm{Au}+\mathrm{Au}$ reactions at the top RHIC energy $(\sqrt{s}=200 \mathrm{AGeV})$, the $C_{B S}$ correlation is constant for all centralities and compatible with the pp result. With increasing width of the rapidity window, $C_{B S}$ follows roughly the shape of the baryon rapidity distribution. We suggest to study the energy and centrality dependence of $C_{B S}$ which allow to gain information on the onset of the deconfinement transition in temperature and volume.
\end{abstract}

Several observables [1] have been proposed throughout the last decades to study the characteristics of the highly excited matter created in heavy ions collisions, where a Quark-Gluon Plasma (QGP) is believed to be created. Among these observables, that give the opportunity to probe whether or not the system went through a phase of deconfined quarks and gluons, the ones related to fluctuations and correlations seem to be the most prospective. Fluctuation probes might be more adequate for the exploration of heavy ion reactions, because the distributions of energy density or initial temperature, isospin and particle density have strong fluctuations from event to event [2, 3, 4]. On the theoretical side event-by-event fluctuations where suggested to study

- kinetic and chemical equilibration in nuclear collisions [5, 6, 7, 8, 9, 10, 11, 12, 13, 14,

- the onset of the deconfinement phase 15, 16, 17, 18, 19, 20, 21.

- the location of the tri-critical end-point of the QCD phase transition [22, 23, 24] or

- the formation of exotic states, like DCCs 25].

On the experimental side, progress has been made by many experiments to extract momentum and particle number ratio fluctuations from heavy ion reaction: Eby-E fluctuations are actively studied in the SPS energy regime (starting from $20 \mathrm{AGeV}$ on) by the NA49 group [26, 27, 28, 29, 30, 31, 32, 33, 34] and the CERES collaboration 35, 36, 37, 38]. At RHIC energies the PHENIX [39, 40, 41] and STAR [42, 43, 44] experiments are addressing the field of single event physics.

Recently a novel event-by-event observable has been introduced by Koch et al. 45, the baryon-strangeness correlation coefficient $C_{B S}$. This correlation is proposed as a tool to specify the nature (ideal QGP or strongly coupled QGP or hadronic matter) of the highly compressed and heated matter created in heavy ions collisions. The idea is that depending on the phase the system is in, the relation between baryon number and strangeness will be different: On the one hand, if one considers an ideal plasma of quarks and gluons, strangeness will be carried by freely moving strange and anti-strange quarks, carrying baryon number in strict proportions. This leads to a strong correlation between the baryon number and strangeness. On the other hand, if the degrees of freedom are of hadronic nature, this correlation is different, because it is possible to carry strangeness without baryon number, e.g. in mesons or QGP bound states.

To quantify to which degree strangeness and baryon number are correlated, the following correlation coefficient has been proposed 45]:

$$
C_{B S}=-3 \frac{\langle B S\rangle-\langle B\rangle\langle S\rangle}{\left\langle S^{2}\right\rangle-\langle S\rangle^{2}}
$$

where $B$ is the baryon charge and $S$ is the strangeness. If a QGP is created, the expected value of $C_{B S}$ will be unity as expected from lattice QCD, compatible with the ideal weakly coupled QGP. In the case of a hadron gas, where the correlation is non trivial, this quantity has been evaluated in [45] to be $C_{B S}=0.66$.

In this paper, we study the correlation coefficient $C_{B S}$ with the Ultra-relativistic Quantum Molecular Dynamics model (UrQMD v2.2). The UrQMD is a nonequilibrium microscopic transport model that simulates the full space-time evolution of heavy ions collisions. It is valid from a few tens of $\mathrm{MeV}$ to several $\mathrm{TeV}$ per nucleons in the laboratory frame. It describes the rescattering of incoming and produced particles, the excitation and fragmentation of color strings and the formation and decay of resonances. This model has been used before to study event-by-event fluctuations rather successfully [4, 7, 16, 21, 25] and yields a reasonable description of inclusive particle distributions. For a complete review of the model, the reader is referred to [46, 47]. 


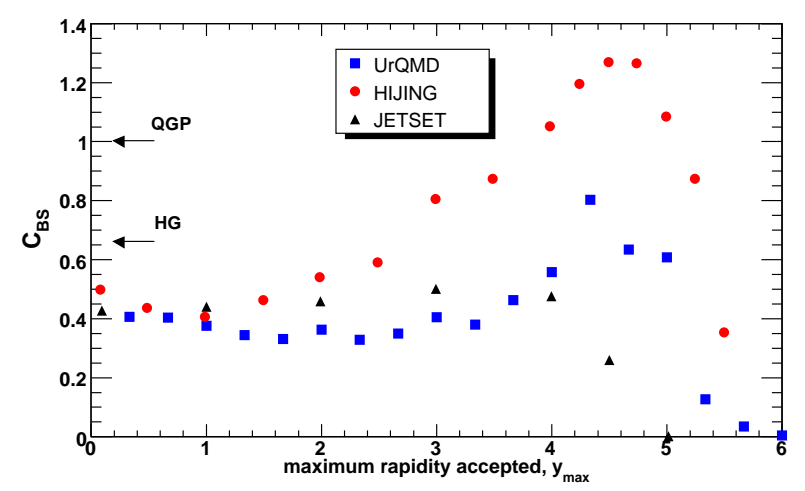

Figure 1: Correlation coefficient for central $\mathrm{Au}+\mathrm{Au}$ collisions at $\sqrt{s}=200$ shown as a function of the maximum rapidity accepted. Circles are the calculation with HIJING [4]]. Squares are the result of the UrQMD calculation and triangles of the JETSET for $e^{+} e^{-}$at $\sqrt{s}=200$. The arrows are the values of a quark gluon plasma and of an hadron gas at a temperature $T=170$ and chemical potential $\mu_{B}=0$ 45]. Both HIJING and JETSET results are taken from [45].

Since the UrQMD is based on hadrons and strings it provides an estimate of the $C_{B S}$ value in the case where no QGP is created, however taking into account the rescattering and the non-equilibrium nature of the heavy ion reactions. $C_{B S}$ is evaluated from the event-byevent fluctuation analyses following [45, 48]:

$$
C_{B S}=-3 \frac{\frac{1}{N} \sum_{n} B^{(n)} S^{(n)}-\left(\frac{1}{N} \sum_{n} B^{(n)}\right)\left(\frac{1}{N} \sum_{n} S^{(n)}\right)}{\frac{1}{N} \sum_{n}\left(S^{(n)}\right)^{2}-\left(\frac{1}{N} \sum_{n} S^{(n)}\right)^{2}}
$$

$B^{(n)}$ and $S^{(n)}$ stand for the baryon number and strangeness in a given event $n$.

The correlation coefficient $C_{B S}$ is depicted in Fig. 1] as a function of the maximum rapidity accepted $(|y| \leq$ $\left.y_{\max }\right)$. The analyzed sample consists of central $\mathrm{Au}+\mathrm{Au}$ events at $\sqrt{s}=200 \mathrm{AGeV}$. For small acceptance windows around midrapidity, $C_{B S}$ stays roughly constant. While for a large acceptance window, $C_{B S}$ increases due to the inclusion of the fragmentation region with high baryon density. The different models deviate from each other for large acceptances due to differences in the handling of the fragmentation region, with small rapidity acceptance (relevant for the RHIC experiments), HIJING, JETSET and UrQMD yield consistent results. If the window acceptance covers all produced particles, $C_{B S}$ has to vanish because of baryon number conservation.

In case a QGP is created, the signal given by the $C_{B S}$ coefficient should survive the hadronic phase. With a strong enough longitudinal flow, strangeness and baryon number within a given rapidity range should be frozen in. The used rapidity window can not be too wide in order to avoid global baryon number and strangeness conservation. Nevertheless, the acceptance window must be wide

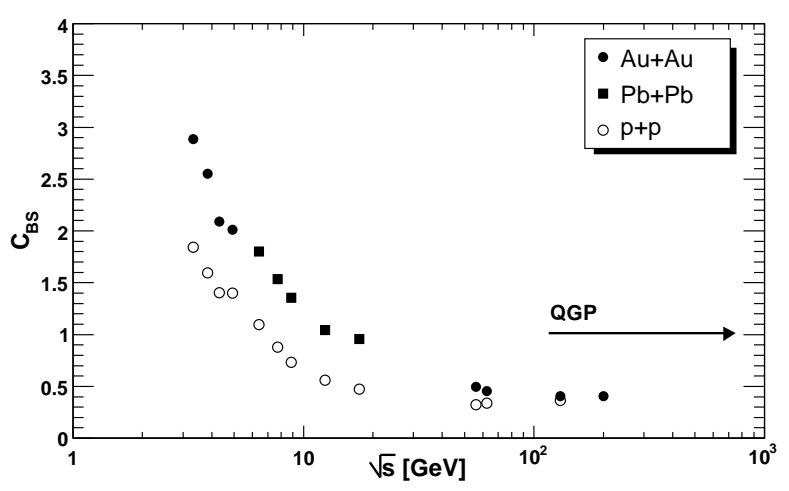

Figure 2: Correlation coefficient $C_{B S}$ for central $\mathrm{Au}+\mathrm{Au} / \mathrm{Pb}+\mathrm{Pb}$ (full symbols) and minimum bias $p+p$ collisions (open symbols) as a function of $\sqrt{s}$. The maximum rapidity accepted is $y_{\max }=0.5$.

enough to avoid smearing due to hadronization.

Figure 2 depicts the energy excitation function of $C_{B S}$ in both $\mathrm{p}+\mathrm{p}$ and centrals $\mathrm{Au}+\mathrm{Au} / \mathrm{Pb}+\mathrm{Pb}$ collisions. As discussed in 45, $C_{B S}$ increases with an increase of the baryon chemical potential $\mu_{B}$ when going to lower beam energies. With increasing collision energy, and therefore decreasing $\mu_{B}, C_{B S}$ goes down to $C_{B S} \approx 0.4$ at the highest RHIC energy available. Surprisingly, the general trend is the same for both $\mathrm{p}+\mathrm{p}$ and $\mathrm{Au}+\mathrm{Au} / \mathrm{Pb}+\mathrm{Pb}$. Measuring the energy dependence of $C_{B S}$ correlation around midrapidity might therefore allow to map out the onset of the QGP production.

The dependence of $C_{B S}$ on the number participants is studied in figure 3 The number of participants is determined via the scaled number of $\pi^{-}$'s in $4 \pi$ geometry $\left(N_{\text {part }}=0.2<\pi^{-}>\right.$). This quantity is proportional to the overlap volume of the colliding nuclei and thus to the number of participants. The UrQMD model predicts a flat dependence of $C_{B S}$ on centrality. $C_{B S} \approx 0.4$ from $p+p$ to central $A u+A u$ events. This is in strong contrast with what is to be expected if the system enters a QGP phase at some centrality. In this case $C_{B S}$ will increase (whether linearly or as a step function depends on the onset behaviour of the QGP phase) from peripheral AA or pp towards central AA collisions. This might allow to extract in detail the volume dependence of the deconfinement transition at RHIC.

To summarize, we have studied the dependence of the baryon-strangeness correlation coefficient as a function of the center of mass energy from $E_{l a b}=4 \mathrm{AGeV}$ to $\sqrt{s}=200 \mathrm{AGeV}$ for pp and central $\mathrm{Au}+\mathrm{Au} / \mathrm{Pb}+\mathrm{Pb}$ reactions. At $\sqrt{s}=200 \mathrm{AGeV}$ we have explored the centrality dependence of the $C_{B S}$ correlation. $C_{B S}$ is found to decrease from the lower energies towards the top RHIC energy available (here $C_{B S} \approx 0.4$ ). For minimum bias $A u+A u$ events at $\sqrt{s}=200$, we predict a flat centrality dependence of $C_{B S}$ near midrapidity. At the highest 


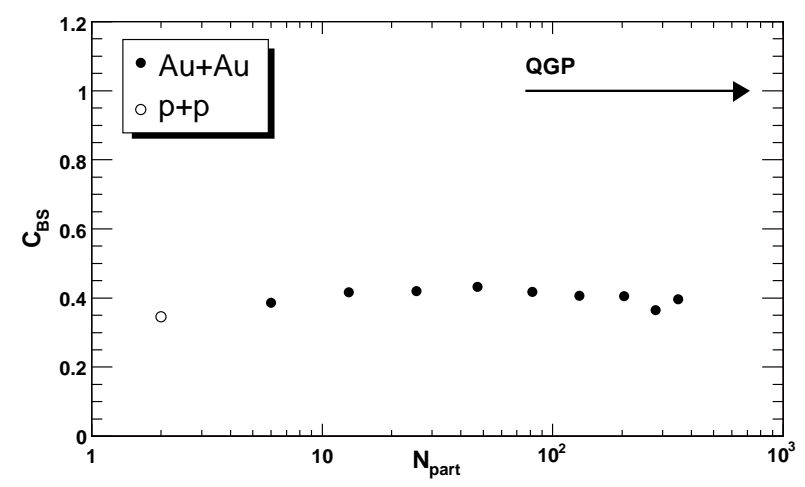

Figure 3: Correlation coefficient for $\mathrm{Au}+\mathrm{Au}$ collisions at $\sqrt{s}=200$ as a function of the number of participants. The maximum rapidity accepted is $y_{\max }=0.5$. Full symbols are the results for $\mathrm{Au}+\mathrm{Au}$ and the open symbol shows the $\mathrm{p}+\mathrm{p}$ value.
RHIC energy the $C_{B S}$ value from the microscopic transport model is roughly half the one expected in the case of a QGP. We suggest to study the energy and centrality dependence of $C_{B S}$ which allow to gain information on the onset of the deconfinement transition in temperature and volume. The CERES/NA49 and STAR experiments should be able to perform these analysis with their accumulated data.

\section{Acknowledgements}

This work is supported by GSI and BMBF.
[1] S. A. Bass, M. Gyulassy, H. Stoecker and W. Greiner, J. Phys. G 25, R1 (1999)

[2] L. Stodolsky, Phys. Rev. Lett. 75 (1995) 1044.

[3] E. V. Shuryak, Phys. Lett. B 423 (1998) 9 arXiv:hep-ph/9704456.

[4] M. Bleicher et al., Nucl. Phys. A 638 (1998) 391.

[5] M. Gazdzicki and S. Mrowczynski, Z. Phys. C 54 (1992) 127.

[6] S. Mrowczynski, Phys. Lett. B 430 (1998) 9 arXiv:nucl-th/9712030.

[7] M. Bleicher et al., Phys. Lett. B 435 (1998) 9 arXiv:hep-ph/9803345.

[8] M. Belkacem, Z. Aouissat, M. Bleicher, H. Stoecker and W. Greiner, arXiv:nucl-th/9903017

[9] S. Mrowczynski, Phys. Lett. B 459 (1999) 13 arXiv:nucl-th/9901078.

[10] A. Capella, E. G. Ferreiro and A. B. Kaidalov, Eur. Phys. J. C 11 (1999) 163 arXiv:hep-ph/9903338.

[11] S. Mrowczynski, Phys. Lett. B 465 (1999) 8 arXiv:nucl-th/9905021.

[12] B. H. Sa, X. Cai, A. Tai and D. M. Zhou, Phys. Rev. C 66 (2002) 044902 arXiv:nucl-th/0112038.

[13] A. Z. Mekjian, arXiv:nucl-th/0411063

[14] S. Mrowczynski, M. Rybczynski and Z. Wlodarczyk, Phys. Rev. C 70 (2004) 054906 arXiv:nucl-th/0407012.

[15] M. Asakawa, U. W. Heinz and B. Muller, Phys. Rev. Lett. 85 (2000) 2072 arXiv:hep-ph/0003169.

[16] M. Bleicher, S. Jeon and V. Koch, Phys. Rev. C 62 (2000) 061902 arXiv:hep-ph/0006201.

[17] B. Muller, Nucl. Phys. A $702 \quad$ (2002) 281 arXiv:nucl-th/0111008.

[18] V. Koch, M. Bleicher and S. Jeon, Nucl. Phys. A 698 (2002) 261 [Nucl. Phys. A 702 (2002) 291] arXiv:nucl-th/0103084.

[19] S. y. Jeon and V. Koch, arXiv:hep-ph/0304012

[20] L. j. Shi and S. y. Jeon, arXiv:hep-ph/0503085

[21] S. Jeon, L. Shi and M. Bleicher, arXiv:nucl-th/0506025

[22] M. A. Stephanov, K. Rajagopal and E. V. Shuryak, Phys.
Rev. Lett. 81 (1998) 4816 arXiv:hep-ph/9806219.

[23] M. A. Stephanov, K. Rajagopal and E. V. Shuryak, Phys. Rev. D 60 (1999) 114028 arXiv:hep-ph/9903292.

[24] Y. Hatta and M. A. Stephanov, Phys. Rev. Lett. 91 (2003) 102003 [Erratum-ibid. 91 (2003) 129901] arXiv:hep-ph/0302002.

[25] M. Bleicher, J. Randrup, R. Snellings and X. N. Wang, Phys. Rev. C 62 (2000) 041901 arXiv:nucl-th/0006047.

[26] J. G. Reid [NA49 Collaboration], Nucl. Phys. A 661 (1999) 407.

[27] H. Appelshauser et al. [NA49 Collaboration], Phys. Lett. B 459 (1999) 679 arXiv:hep-ex/9904014.

[28] S. V. Afanasev et al. [NA49 Collaboration], Phys. Rev. Lett. 86 (2001) 1965 arXiv:hep-ex/0009053.

[29] C. Alt et al. [NA49 Collaboration], Phys. Rev. C 70 (2004) 064903 arXiv:nucl-ex/0406013.

[30] C. Roland et al. [NA49 Collaboration], J. Phys. G 30 (2004) S1381 arXiv:nucl-ex/0403035.

[31] T. Anticic et al. [NA49 Collaboration], Phys. Rev. C 70 (2004) 034902 arXiv:hep-ex/0311009.

[32] C. Alt et al. [NA49 Collaboration], Phys. Rev. C 71 (2005) 034903 arXiv:hep-ex/0409031.

[33] M. Rybczynski et al. [NA49 Collaboration], J. Phys. Conf. Ser. 5 (2005) 74 arXiv:nucl-ex/0409009.

[34] C. Roland [NA49 Collaboration], J. Phys. G 31 (2005) S1075.

[35] D. Adamova et al. [CERES Collaboration], Nucl. Phys. A 727 (2003) 97 arXiv:nucl-ex/0305002.

[36] H. Appelshauser [CERES Collaboration], Prog. Part. Nucl. Phys. 53 (2004) 253.

[37] H. Sako and H. Appelshaeuser [CERES/NA45 $\begin{array}{lllllll}\text { Collaboration], J. Phys. G } & \mathbf{3 0} & \text { (2004) } & \text { S1371 }\end{array}$ arXiv:nucl-ex/0403037.

[38] H. Appelshauser et al. [CERES Collaboration], Nucl. Phys. A 752 (2005) 394 arXiv:nucl-ex/0409022.

[39] K. Adcox et al. [PHENIX Collaboration], Phys. Rev. Lett. 89 (2002) 082301 arXiv:nucl-ex/0203014.

[40] K. Adcox et al. [PHENIX Collaboration], Phys. Rev. C 
66 (2002) 024901 arXiv:nucl-ex/0203015.

[41] S. S. Adler et al. [PHENIX Collaboration], Phys. Rev. Lett. 93 (2004) 092301 arXiv:nucl-ex/0310005.

[42] J. Adams et al. [STAR Collaboration], Phys. Rev. C 68 (2003) 044905 arXiv:nucl-ex/0307007.

[43] J. Adams et al. [STAR Collaboration],

[44] J. Adams et al. [STAR Collaboration], arXiv:nucl-ex/0406035

[45] V. Koch, A. Majumder and J. Randrup, arXiv:nucl-th/0505052
[46] S. A. Bass et al., Prog. Part. Nucl. Phys. 41 (1998) 225 arXiv:nucl-th/9803035.

[47] M. Bleicher et al., J. Phys. G 25 (1999) 1859 arXiv:hep-ph/9909407.

[48] Note that this relation has an additional factor -3 which was accidentally omitted in the pre-print [45]. (J. Randrup, private communication, 2005)

[49] X. N. Wang and M. Gyulassy, Phys. Rev. D 44 (1991) 3501. 